\title{
Contralateral tumor seeding of renal cell carcinoma mimicking late metastasis of liver after laparoscopic nephrectomy: A case report with review of the literature
}

\author{
Özgür Haki Yüksel, Caglar Yildirim, Ahmet Ürkmez, Serkan Akan, Ayhan Verit \\ Fatih Sultan Mehmet Research \& Training Hospital, Dept. of Urology, Istanbul, Turkey.
}

\begin{abstract}
Summary Laparoscopic surgery has been increasingly used every day in the management of urologic malignancies. Even though it seems as a minimally invasive surgery, during these interventions tumor seeding, as seen in open surgery, confronts us as a rarely seen serious risk. Herein, we have reported a case who demonstrated peritoneal tumor implantation at 12 month postoperative follow up after laparoscopic radical nephrectomy (LRN) performed for Furhman grade 1 (T2NOMO) renal cell cancer.
\end{abstract}

KEY WORDS: Laparoscopic radical nephrectomy; Tumor seeding; Peritoneal implant; Renal cell cancer.

Submitted 9 March 2015; Accepted 30 April 2015

\section{INTRODUCTION}

During removal of malignant tumours by means of open surgery or laparoscopic resection, direct inoculation of neoplastic cells can occur. This inoculation is generally seen along the port access route which is known as portsite metastasis (1). In laparoscopic surgeries applied for the management of urologic malignancies, peritoneal implants or port-site metastases have been rarely (0.09$0.03 \%$ ) seen up to now (2). Although etiological factors of this phenomenon have not been completely understood, tumor aggressivity, laparoscopy-related factors, immune system of the patient and local characteristics of the surgical site have been implicated. Among all urologic malignancies, peritoneal tumor seeding and portsite metastasis are extremely rarely seen in renal cell cancer (RCC). Very scarce number of case reports have been reported in the literature (3). Herein, we presented a case who demonstrated peritoneal tumor implantation at postoperative 12 month follow up after laparoscopic radical nephrectomy (LRN) performed for Furhman grade 1 (T2NOMO) renal cell cancer (RCC).

\section{Case report}

A 68-year-old male patient consulted to our outpatient clinic in December 2012 with complaints of dysuria and pollakiuria. His medical evaluation revealed a high serum PSA level $(10.5 \mathrm{ng} / \mathrm{ml})$ which necessitated prosta- tic biopsy with the aid of transrectal ultrasound. Histopathological report indicated prostate cancer (PCa) with Gleason score 3+3 in 6 out of 12 core biopsy specimens. Magnetic resonance imaging (MRI) performed for tumor grading incidentally disclosed a mass lesion radiologically suggesting RCC located in the mid-portion of the left kidney with exophytic extension and measuring $45 \mathrm{~mm}$ in its largest diameter. With this indication, the patient underwent laparoscopic renal nephrectomy (LRN) in December 2012. His histopathological report indicated the lesion to be Furhman grade 1 (T2NOMO) RCC (Figure 1). Afterwards, we performed radical retropubic prostatectomy and lymph node dissection with the indication of PCa in March 2013 and penile prosthesis was implanted to treat his erectile dysfunction. Up to that time, laboratory and radiological controls did not reveal the presence of RCC and PCa recurrences or metastases.

Control MRI performed at 12 month follow up postLRN, disclosed a perihepatic mass lesion with dimensions of $20 \times 18 \times 12 \mathrm{~mm}$ consistent with metastasis or tumor seeding located on the anterior segment of the right lobe of the liver (Figure 2). Then, the patient underwent laparotomy in February 2014 in the department of general surgery and the lesion visualized during MRI was revealed to be a peritoneal tumor implant. The implant was excised and consulted during the perioperative period to the department of histopathology for frozen section. Histopathological report indicated the lesion to be a RCC implant (Figure 3). The patient is still at post-LRN 24. months. All abdominal MRI, thoracic $\mathrm{CT}$ and bone scans could not reveal any evidence of clinically significant RCC and PCa recurrence or metastases.

\section{Conclusions}

In conclusion, following oncologic laparoscopic interventions, not only local recurrence and distant metastases, but also peritoneal implantation should be also considered and atypical abdominal small lesions away from trocar access site should be also taken into consideration as for implantation metastases.

Discussion and supplementary references are posted in Supplementary Materials on www.aiua.it 


\section{Figure 1.}

Hematoxylin and eosin stain of the left kidney: Renal cell carcinoma (40X).

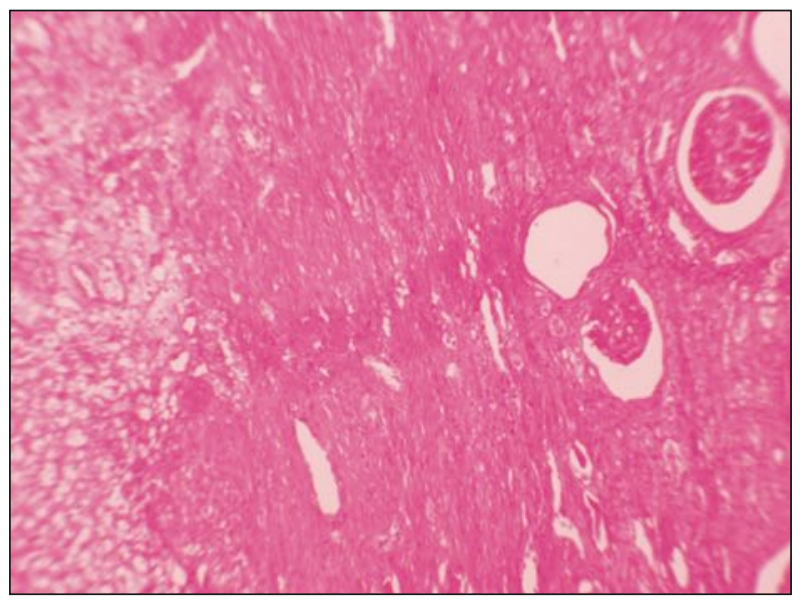

\section{Figure 2.}

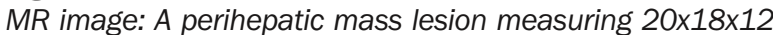
$\mathrm{mm}$ located on the anterior segment of the right lobe of the liver.

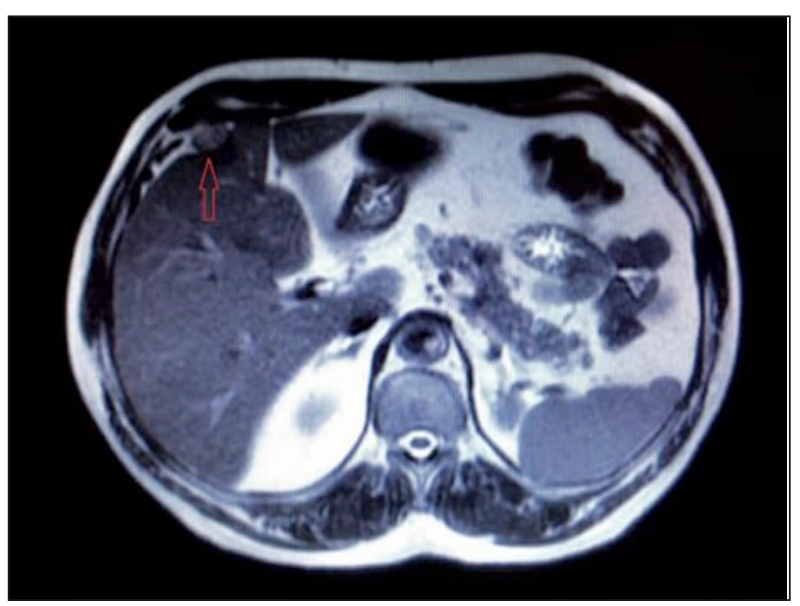

Figure 3.

Hematoxylin and eosin stain of the peritoneal renal cell carcinoma implant (100X).

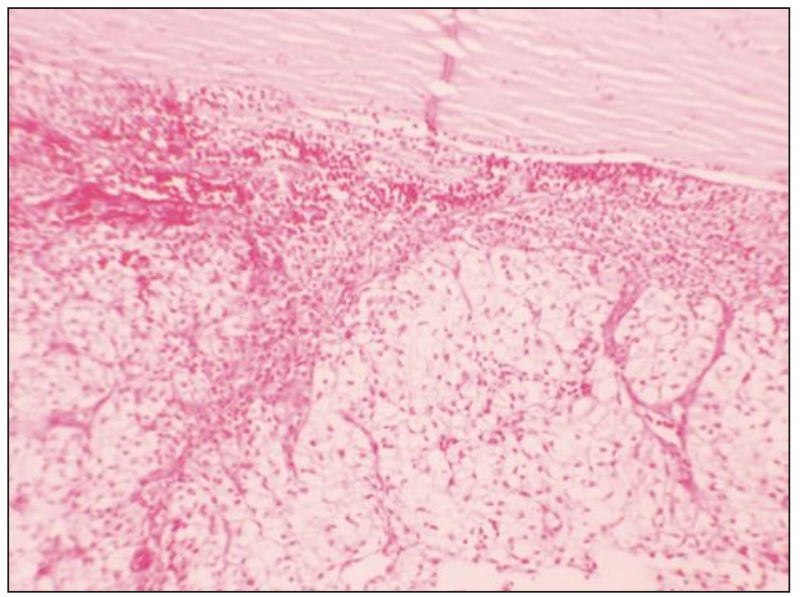

\section{REFERENCES}

1. Lee BR, Tan BJ, Smith AD. Laparoscopic port site metastases: incidence, risk factors, and potential preventive measures. Urology. 2005; 65:639-644.

2. Tanaka K, Hara I, Takenaka A, et al. Incidence of local and port site recurrence of urologic cancer after laparoscopic surgery. Urology. 2008; 71:728-734.

3. Castillo OA, Vitagliano G. Port site metastasis and tumor seeding in oncologic laparoscopic urology. Urology. 2008; 1:372-378.

\section{Correspondence}

Özgür Haki Yüksel, MD (Corresponding Author) ozgurhaki@gmail.com

Caglar Yildirim, MD

Ahmet Ürkmez, MD

Serkan Akan, MD

Ayhan Verit, MD. Prof.

Fatih Sultan Mehmet Research and Training Hospital, Dept. of Urology, Içerenköy/Ataşehir, Tr- 34752 Istanbul, Turkey 